\title{
First proof of association between autoimmune polyglandular syndrome and multiple endocrine neoplasia in humans
}

\author{
Jacopo Manso ${ }^{1)}$, Simona Censi ${ }^{1)}$, Maurizio Iacobone ${ }^{2)}$, Francesca Galuppini ${ }^{3)}$, Gianmaria Pennelli ${ }^{3)}$, \\ Corrado Betterle ${ }^{1)}$ and Caterina Mian ${ }^{1)}$ \\ 1) Department of Medicine (DIMED), Endocrinology Unit, Padua University, Padua, Italy \\ 2) Department of Surgical, Oncological and Gastroenterological Sciences (DiSCOG), Endocrine Surgery Unit, Padua University \\ Hospital, Padua, Italy \\ 3) Department of Medicine (DIMED), Surgical Pathology and Cytopathology Unit, Padua University, Padua, Italy
}

\begin{abstract}
Autoimmune Addison's disease (AAD) is a rare condition occurring either in isolation or associated with other autoimmune diseases as part of an autoimmune polyglandular syndrome (APS) type 1, 2 or 4. Multiple endocrine neoplasia (MEN) type 1, 2 or 4 is a hereditary autosomal dominant cancer syndrome. Medullary thyroid carcinoma and pheochromocytoma are neoplasms common to MEN-2a and MEN-2b. We describe a unique, complex case of a man resulted affected by both APS-2 and MEN-2a. The patient developed Hashimoto's thyroiditis, diabetes mellitus type 1 and AAD, despite testing negative for adrenal cortex autoantibodies (ACA) and steroid 21-hydroxylase autoantibodies (21-OHAb). Moreover, he had also a family history for MEN-2a and he first developed medullay thyroid cancer, then bilateral pheochromocytoma on the adrenal substrate of an AAD. On adrenal histology we found complete bilateral cortical atrophy in the presence of a lymphocytic infiltration and fibrosis, confirming an ACA and 21-OHAb-negative AAD. This datum is the first documented in a living individual and confirms that the absence of autoantibodies is not incompatible with an autoimmune disease and confirms that AAD is a cell-mediated autoimmune disease limited to the adrenal cortex and sparing medullary. In the light of a literature review concerning the association between APS and MEN, this is the first proven case to be reported in humans. Finally, our findings suggest that adrenal medullary tumor can develop even on an adrenal gland with cortical atrophy due to autoimmune adrenalitis.
\end{abstract}

Key words: Multiple endocrine neoplasia, Autoimmune polyglandular syndrome, Addison's disease, Medullary thyroid cancer, Pheochromocytoma

PRIMARY adrenocortical insufficiency, or Addison's disease $(\mathrm{AD})$, is a rare life-threatening condition with diverse etiologies $[1,2]$. Autoimmune destruction of the adrenal cortex is currently responsible for the majority of cases in adults. Autoimmune Addison's disease (AAD) can present as an isolated condition or be associated with other autoimmune diseases, forming part of an autoimmune polyglandular syndrome (APS) type 1, 2 or 4 [3-5]. APS-1 is characterized by AAD in association with chronic candidiasis and/or chronic hypoparathyroidism. In APS-2, AAD is associated with autoimmune thyroid diseases and/or diabetes mellitus type 1 (DM-1). APS-4 involves AAD in association with any other auto-

Submitted Feb. 27, 2020; Accepted Apr. 20, 2020 as EJ20-0099 Released online in J-STAGE as advance publication May 29, 2020 Correspondence to: Caterina Mian, M.D., Ph.D., Endocrine Unit, Department of Medicine (DIMED), Università di Padova, Via Ospedale Civile 105, 35128 Padova, Italy.

E-mail: caterina.mian@unipd.it immune diseases (Table 1).

In cases of adrenal cortical insufficiency, the diagnosis of AAD is based on the detection of adrenal cortex autoantibodies (ACA) and/or steroid 21-hydroxylase autoantibodies (21-OHAb). ACA and/or 21-OHAb assays performed within 2 years after the diagnosis of AAD can be positive in about $90 \%$ of cases $[2,6]$.

Multiple endocrine neoplasia (MEN) types 1, 2 and 4 are hereditary autosomal dominant cancer syndromes. MEN-1 is a complex, heterogeneous syndrome caused by germline mutations of the MEN1 gene. It is characterized by the presence of at least two of the three endocrine tumors mainly involved in MEN-1: parathyroid adenomas, pituitary tumors and entero-pancreatic endocrine tumors [7].

MEN-2 is currently divided into types $2 \mathrm{a}$ and type $2 \mathrm{~b}$. It is caused by germline mutations in the RET protooncogene [8, 9]. The typical clinical features of MEN-2 variants are listed in Table 2. Medullary thyroid carcinoma 
Table 1 APS Classification according to Neufeld and Blizzard

\begin{tabular}{ll}
\hline APS-1 Chronic candidiasis, chronic hypoparathyroidism, AAD (at least two conditions must be present) \\
APS-2 $\begin{array}{l}\text { AAD + autoimmune thyroid diseases (Basedow or Hashimoto's thyroiditis) and/or type } 1 \text { diabetes mellitus (AAD must always } \\
\text { be present) }\end{array}$ \\
APS-3 $\begin{array}{l}\text { Thyroid autoimmune diseases (Basedow or Hashimoto's thyroiditis) + other autoimmune diseases (excluding AAD, } \\
\text { hypoparathyroidism, chronic candidiasis) }\end{array}$ \\
APS-4
\end{tabular}

AAD, autoimmune Addison disease; APS, autoimmune polyglandular syndrome.

Table 2 Typical clinical features of MEN-2 variants

\begin{tabular}{|c|c|c|}
\hline \multirow{4}{*}{ MEN-2a } & classical & $\begin{array}{l}\text { MTC } \\
\text { PHEO } \\
\text { PHPTH (hyperplasia, adenoma, carcinoma) }\end{array}$ \\
\hline & with Hirschsprung's disease & MEN-2a + Hirschsprung's disease \\
\hline & with cutaneous lichen amyloidosis & MEN-2a + pruritic cutaneous lesion located over the upper back (lichen amyloidosis) \\
\hline & FMTC & Presence of RET germline mutation in families who develop neither PHEO nor PHPTH \\
\hline MEN-2b & & $\begin{array}{l}\text { MTC } \\
\text { PHEO } \\
\text { ganglioneuromas } \\
\text { marfanoid habitus }\end{array}$ \\
\hline
\end{tabular}

FMTC, familial medullary thyroid carcinoma; MEN, multiple endocrine neoplasia; MTC, medullary thyroid carcinoma; PHEO, pheochromocytoma; PHPTH, primary hyperparathyroidism.

(MTC) and pheochromocytoma (PHEO) are neoplasms common to both MEN-2a and MEN-2b. MEN-4 is defined as a condition resembling a MEN-1 syndrome in patients with no MEN1 gene mutations; $C D K N 1 B$ gene (encoding p27) mutations have been found in some cases [10].

In the case described here, we wish to emphasize the association of APS-2 with MEN-2a in the same patient. To the best of our knowledge, this is the first case of such an association in humans to be reported in the literature.

\section{Case Report}

In 1987, a 25-year-old male presented with a history of some months of asthenia, weight loss, hypotension, low-grade fever $\left(37.5-38^{\circ}\right)$, and diffuse myalgia. The patient had a family history including: MEN-2a in a sister, an aunt and a first-degree maternal cousin; diabetes mellitus type 2 and ischemic heart disease in his father; and his mother had died at 31 years old of unknown causes. Laboratory tests to assess the pituitary-adrenal axis showed: elevated basal adrenocorticotropic hormone (ACTH) at 8 am (275 pmol/L, normal range [n.r.] 10-50 $\mathrm{pmol} / \mathrm{L})$; reduced morning serum cortisol (11 nmol/L, n.r. $138-690 \mathrm{nmol} / \mathrm{L})$; undetectable serum levels of dehydroepiandrosterone sulfate; high blood potassium (5.4 mmol/L, n.r. $3.5-5 \mathrm{mmol} / \mathrm{L}$ ) and low blood sodium
(128 mmol/L n.r. 135-145 mmol/L) levels; low 24-h free urinary cortisol (10 nmol/24 h, n.r. 16-168 nmol/24 h); an augmented orthostatic plasma renin activity (34 $\mathrm{ug} / \mathrm{L} / \mathrm{h}$, n.r. $0.6-4.8 \mathrm{ug} / \mathrm{L} / \mathrm{h}$ ); and no cortisol response at $60 \mathrm{~min}$ to an intravenous dose of $250 \mu \mathrm{g}$ ACTH (11 $\mathrm{nmol} / \mathrm{L}$, n.r. $>500 \mathrm{nmol} / \mathrm{L})$. Other hormone assays showed high thyrotropin (6.9 mIU/L, n.r. $0.20-4 \mathrm{mIU} / \mathrm{L})$ with blood free-thyroxine and free-triiodothyronine, fasting blood glucose and glycated hemoglobin in the normal range; plasma calcitonin (CT) values were not available. Autoantibody screening revealed the presence of autoantibodies to thyroperoxidase (TPOAbs), islet cells (ICA), glutamate decarboxylase (GADAbs), and insulin (IAA), while no ACA or 21-OHAb were found. Thyroid ultrasound was compatible with Hashimoto's thyroiditis with no nodular lesions. The adrenal glands appeared smaller than normal, but with a regular morphology on computed tomography. A diagnosis of $\mathrm{AD}$ and Hashimoto's thyroiditis with autoantibodies to endocrine pancreas was established. Although the patient tested negative for ACA and 21-OHAb, given his normal adrenal glands, Hashimoto's thyroiditis and pancreatic autoimmunity, the AD was considered an AAD and so the patient was diagnosed with APS-2. The search for other autoimmune diseases that can potentially develop less frequently in APS-2-i.e. hypergonadotropic hypogonadism, vitiligo, alopecia, chronic hepatitis, chronic atrophic gastritis and hypophysitis - has produced no 


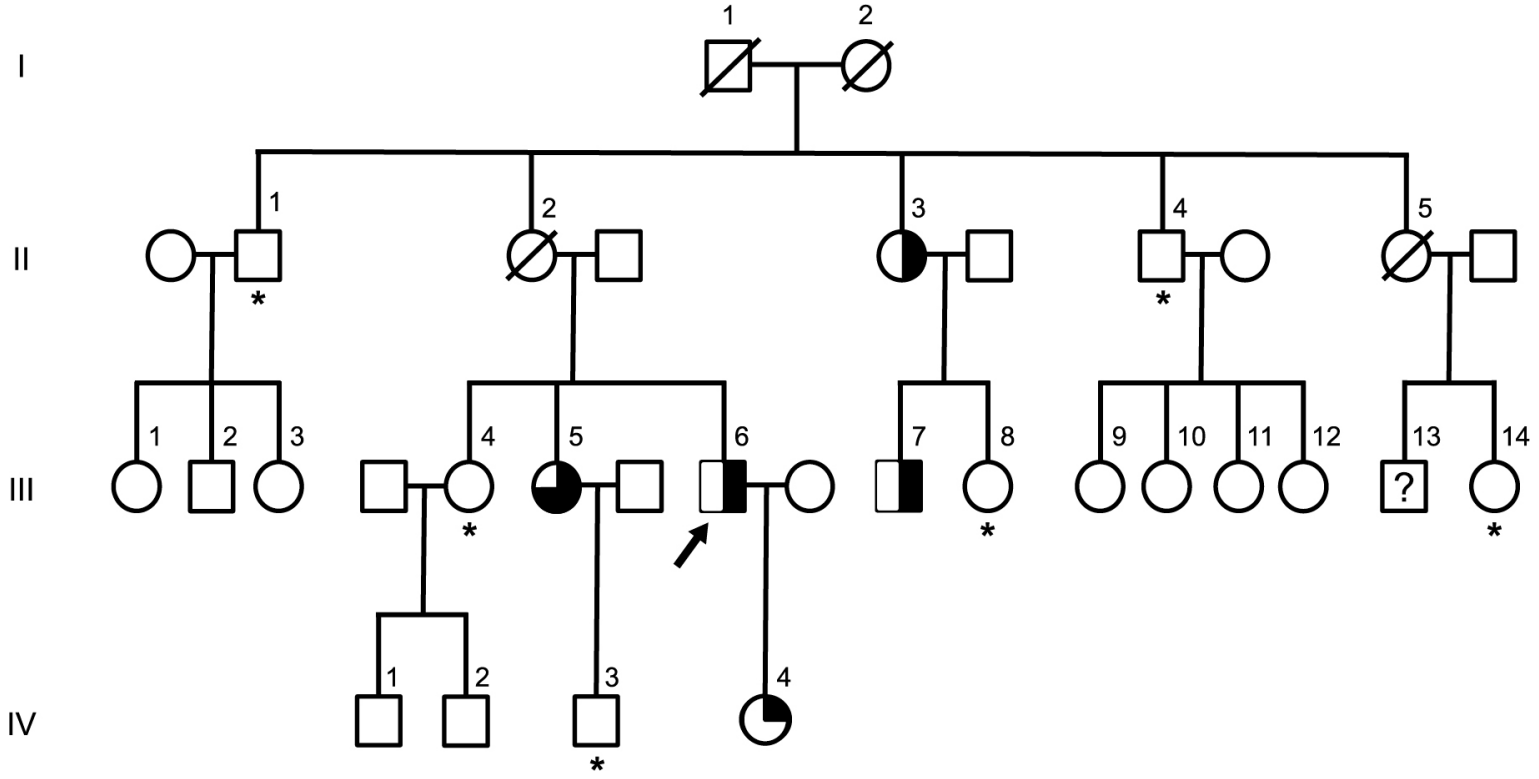

Fig. 1 Pedigree analysis. The proband is indicated by a black arrow. Deceased patients are identified by a diagonal line. Subjects with signs of MEN2A syndrome are indicated by black shading, which covers a quarter of the pedigree symbol for patients with MTC alone, half of the symbol for patients with MTC plus PHEO, and three-quarters of the symbol for MTC plus PHEO plus primary hyperparathyroidism. All patients who underwent genetic testing are marked with an asterisk. After genotyping, wild type RET carriers are identified by an "asterisk" sign in their respective pedigree symbols.

results. Replacement therapy with cortisone acetate and fludrocortisone was started with clinical benefit. No thyroid replacement therapy was initiated because the patient remained in the range of subclinical hypothyroidism in the following years.

In 1996, at the age of 34, the patient was referred for the first time to our Endocrine Unit because of a thyroid nodule in the right lobe and high levels of plasma CT (1.176 ng/mL, n.r. $0-0.130 \mathrm{ng} / \mathrm{mL}$ ), and carcinoembryonic antigen (CEA) (26.4 ng/L, n.r. 0-4 ng/L). After MTC was confirmed by fine needle aspiration biopsy, the patient underwent total thyroidectomy with level VI lymph node dissection. 24-h urinary catecholamines were in the normal range before surgery. Histology confirmed the presence of MTC in a background of autoimmune thyroiditis, and final pathological staging was T1N0M0 (according to the 8th edition of the American Joint Committee on Cancer TNM staging system [11]) in a context of autoimmune thyroiditis. Plasma CT and CEA dropped to negative values (CT 0.049 ng/L, CEA $0.5 \mathrm{ng} / \mathrm{mL}$ ). Given the patient's positive family history for MEN-2a, a genetic analysis was conducted on the RET gene, which identified a germline mutation on exon 11, Cys634Gly. We reported the pedigree of the family in Fig. 1. At the latest oncological follow-up, the patient's MTC was still in remission and there is no evidence of primary hyperparathyroidism.

In 2003, aged 41, the patient developed a clinical autoimmune diabetes mellitus type 1 , confirmed by the pres- ence of GADAbs, thus configuring a complete APS-2. An insulin micro-pump was inserted in 2015.

In 2017, at 55 years old, a routine check-up on the MEN-2a syndrome showed high 24-h urinary metanephrines (4.32 $\mu \mathrm{mol} / 24 \mathrm{~h}$, n.r. $0.01-1.62 \mu \mathrm{mol} / 24 \mathrm{~h})$ and normetanephrines $(2.84 \mu \mathrm{mol} / 24$ h, n.r. $0.01-2.13$ $\mu \mathrm{mol} / 24 \mathrm{~h}$ ), but no hypertension or other clinical symptoms. Indeed, the previous 24-h urinary metanephrines and normetanephrines during annual follow-up were in normal ranges (metanephrines $1.43 \mathrm{umol} / 24 \mathrm{~h}$ and normetanephrines $1.04 \mathrm{umol} / 24 \mathrm{~h}$ ). A contrast-enhanced magnetic resonance scan of the abdomen showed a solid expansive mass in the right adrenal loggia with clear margins, $3.9 \times 2.9 \times 3.6 \mathrm{~cm}$ in size, with a signal predominantly hyper-intense on T2-weighted, and iso-/ hypo-intense on T1-weighted images, which were amply inhomogeneous. The scan also showed a rapid, inhomogeneous arterial enhancement with a rapid wash-out in the venous phase. The left adrenal showed a similar lesion around $3 \mathrm{~cm}$ in size. The patient consequently underwent surgery after adequate preparation with alphablockers because of a strong suspicion of bilateral adrenal PHEO in MEN-2a. Histology confirmed bilateral widespread PHEO of the adrenal gland with a low mitotic index, and concomitant bilateral cortical atrophy with lymphocytic infiltrate compatible with autoimmune adrenalitis (AAD), as shown in Fig. 2. After surgery, the oncological follow-up showed normalization of the metanephrine levels and disease remission. 


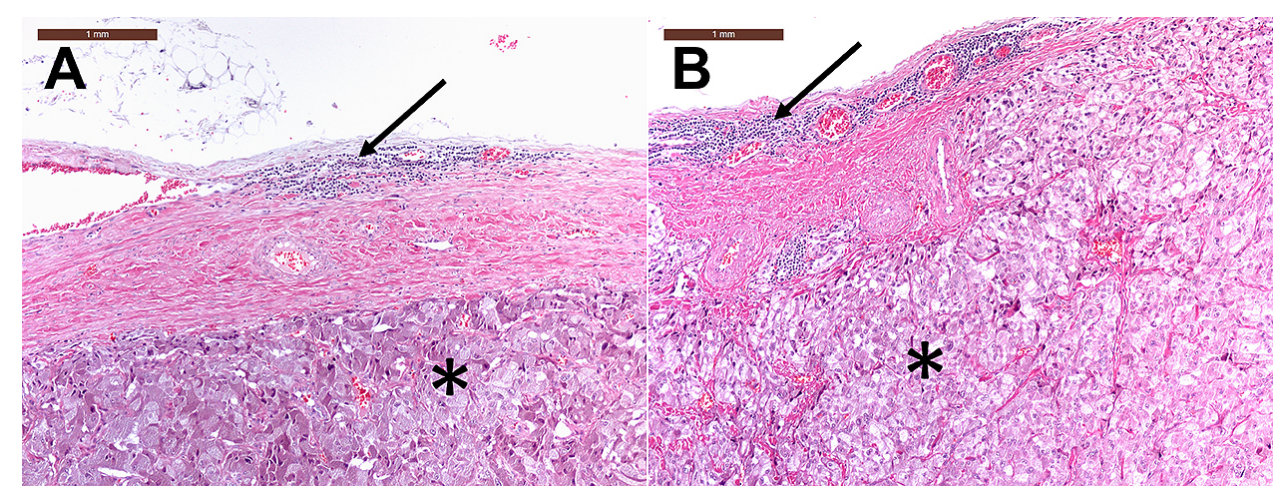

Fig. 2 A: Histopathology of the right adrenal gland: medullary pheochromocytoma (*) and concomitant cortical atrophy with lymphocytic infiltrate and fibrosis (autoimmune adrenalitis) (arrow); B: Histopathology of the left adrenal gland: medullary pheochromocytoma $(*)$ and concomitant cortical atrophy with lymphocytic infiltrate and fibrosis (autoimmune adrenalitis) (arrow).

\section{Discussion}

The above clinical scenario paints a unique and particularly complex picture. To the best of our knowledge, it is the first case report in the literature of APS and MEN occurring in the same person.

The patient was initially diagnosed with APS-2 $[2,4]$ and $\mathrm{AAD}$, despite testing negative for ACA and 21OHAb. Diagnosis of AAD, in the presence of adrenal cortical insufficiency, is based on the detection of ACA and/or 21-OHAb; however, 21-OHAb are not always present in the AAD but approximately in the $78 \%, 91 \%$ and $75 \%$ of patients with APS type 1, type 2 and isolated $\mathrm{AD}$, respectively [2]. Our case falls into the category of AAD without ACA and 21-OHAb positivity (possible in $9 \%$ of patients with APS type 2). On adrenal histology performed 21 years later we found complete bilateral cortical atrophy in the presence of a lymphocytic infiltration and fibrosis, confirming an ACA- and 21-OHAbnegative AAD in this patient. This datum is the first documented in a living individual and confirms that the absence of autoantibodies is not incompatible with an autoimmune disease and confirms that AAD is a cellmediated autoimmune disease limited to the adrenal cortex and sparing medullary with an HLA predisposition.

Histology also vouched the patient's MEN-2a: he first developed MTC, then bilateral PHEO on the adrenal substrate of an AAD. Reviewing the literature, we found only a few cases of a possible, but not proven association between APS and MEN. A first report in 1998 describes a patient with primary hypothyroidism, mucocutaneous candidiasis and vitiligo (a possible case of APS-1) associated with a Cushing syndrome and primary hyperparathyroidism (possible MEN-1) [12]. The authors can only suggest such a diagnosis, however, because there are no other signs of APS-1 (AAD and chronic hypoparathyr- oidism), and no genetic analyses were conducted to confirm germline mutations of the AIRE and MEN1 genes.

In 2008, another case report was published that describes a Spanish patient previously diagnosed with APS-2 developing unilateral PHEO [13]. Indeed, in this case we are in the context of a sporadic pheochromocytoma arising on an atrophic adrenal gland because of the autoimmune process. Unlike other causes of $\mathrm{AD}$, the autoimmune form (AAD) does not produce the total destruction of the adrenal gland; the adrenalitis only affects the cortical part, while the medulla is preserved. This does not exclude the possibility of developing a tumor originating from the medulla, such as PHEO. Curiously, in this other unique association of PHEO with $\mathrm{AAD}$ in a human, an ACA assay performed 22 years later was negative - as in our case [13].

Finally, Arias et al. described a dog with AAD that developed bilateral PHEO. After euthanasia, a single parathyroid adenoma (PA) was identified, with a normocalcemic hyperparathyroidism [14]. The authors suggested that the co-presence of bilateral PHEO and PA might configure a particular form of MEN-2a, despite no MTC (which has a $100 \%$ penetrance in humans), but this claim appears difficult to sustain. In fact, the presence of MTC in MEN-2a has been described in dogs as well [15-17] and, despite the occasional finding of a PA, a diagnosis of primary normocalcemic hyperparathyroidism cannot be established without excluding a secondary form due to vitamin $\mathrm{D}$ deficiency (a possibility not investigated by the authors).

In our case, the carcinogenic process in the adrenal medullary tissue was certainly influenced more by the presence of the RET germline mutation that configures MEN-2a than by the autoimmune cortical adrenalitis. We would like to underscore the unprecedented association of MEN-2a and APS-2 in the same patient, with the 
development of a bilateral PHEO in the context of a longstanding AAD. To the best of our knowledge, this the first case to be reported in humans.

Finally, our findings suggest that adrenal medullary tumor can develop even on an adrenal gland with cortical atrophy due to autoimmune adrenalitis. Screening for PHEO should therefore also be considered for patients with AAD who have other risk factors for the onset of adrenal medullary tumors, such as a genetic predisposition.

\section{Acknowledgments}

We thank Frances Coburn for text editing.

\section{Authors' Contributions}

All the authors accept responsibility for the whole content of this manuscript as submitted and approve its submission.

\section{Research Funding}

None to disclose.

\section{Employment or Leadership}

None to disclose.

\section{Honorarium}

None to disclose.

\section{Competing Interests}

None to disclose.

\section{Ethical Conduct of Research}

Informed consent was obtained from the patient involved in this case report.

\section{Disclosure Summary}

The authors have nothing to disclose.

\section{References}

1. Husebye ES, Allolio B, Arlt W, Badenhoop K, Bensing S, et al. (2014) Consensus statement on the diagnosis, treatment and follow-up of patients with primary adrenal insufficiency. J Intern Med 275: 104-115.

2. Betterle C, Dal Pra C, Mantero F, Zanchetta R (2002) Autoimmune adrenal insufficiency and autoimmune polyendocrine syndromes: autoantibodies, autoantigens, and their applicability in diagnosis and disease prediction. Endocr Rev 23: 327-364.

3. Betterle C, Presotto F, Furmaniak J (2019) Epidemiology, pathogenesis, and diagnosis of Addison's disease in adults. J Endocrinol Invest 42: 1407-1433.

4. Manso J, Pezzani R, Scarpa R, Gallo N, Betterle C (2018) The natural history of autoimmune Addison's disease with a non-classical presentation: a case report and review of literature. Clin Chem Lab Med 56: 896-900.

5. Neufeld M, Maclaren NK, Blizzard RM (1981) Two types of autoimmune Addison's disease associated with different polyglandular autoimmune (PGA) syndromes. Medicine (Baltimore) 60: 355-362.

6. Betterle C, Volpato M, Pedini B, Chen S, Smith BR, et al. (1999) Adrenal-cortex autoantibodies and steroidproducing cells autoantibodies in patients with Addison's disease: comparison of immunofluorescence and immunoprecipitation assays. J Clin Endocrinol Metab 84: 618622.
7. Brandi ML, Gagel RF, Angeli A, Bilezikian JP, BeckPeccoz P, et al. (2001) Guidelines for diagnosis and therapy of MEN type 1 and type 2. J Clin Endocrinol Metab 86: 5658-5671.

8. Wells SA, Pacini F, Robinson BG, Santoro M (2013) Multiple endocrine neoplasia type 2 and familial medullary thyroid carcinoma: an update. J Clin Endocrinol Metab 98: 3149-3164.

9. Wells SA, Asa SL, Dralle H, Elisei R, Evans DB, et al. (2015) Revised American Thyroid Association Guidelines for the Management of Medullary Thyroid Carcinoma. Thyroid 25: 567-610.

10. Thakker RV (2014) Multiple endocrine neoplasia type 1 (MEN1) and type 4 (MEN4). Mol Cell Endocrinol 386: 215.

11. Tuttle RM, Haugen B, Perrier ND (2017) Updated American joint committee on cancer/tumor-node-metastasis staging system for differentiated and anaplastic thyroid cancer (Eighth Edition): what changed and why? Thyroid 27: 751-756.

12. Bahl VK, Sandhu A, Mohan V, Nolan S (1998) Multiple endocrine neoplasia and polyglandular autoimmune syndrome: a new association. Endocr Pract 4: 208-212.

13. Toni García M, Anda Apiñániz E, Pablo J, de Esteban M, Munárriz Alcuaz P, et al. (2008) An unusual association: pheochromocytoma on an atrophied adrenal gland due to 
addison's disease. Endocrinol Nutr 55: 510-513.

14. Arias EAS, Castillo VA, Trigo RH (2018) Addison disease and normocalcemic primary hyperparathyroidism in a dog with multiple endocrine neoplasia. Open Vet J 7: 332-336.

15. Arias EAS, Castillo VA, Trigo RH, Caneda Aristarain ME (2016) Multiple endocrine neoplasia similar to human subtype $2 \mathrm{~A}$ in a dog: medullary thyroid carcinoma, bilateral pheochromocytoma and parathyroid adenoma. Open
Vet J 6: 165-171.

16. Wright KN, Breitschwerdt EB, Feldman JM, Berry CR, Meuten DJ, et al. (1995) Diagnostic and therapeutic considerations in a hypercalcemic dog with multiple endocrine neoplasia. J Am Anim Hosp Assoc 31: 156-162.

17. Peterson ME, Randolph JF, Zaki FA, Heath H 3rd (1982) Multiple endocrine neoplasia in a dog. $J$ Am Vet Med Assoc 180: 1476-1478. 